\title{
Methyl Ganoderic Acid DM: A Selective Potent Osteoclastogenesis Inhibitor
}

\author{
Jie Liu $^{1}$, Jun Shiono ${ }^{1}$, Yukiko Tsuji ${ }^{2}$, Kuniyoshi Shimizu, ${ }^{1, *}$ and Ryuichiro Kondo ${ }^{1}$ \\ ${ }^{1}$ Department of Forest and Forest Products Science, Faculty of Agriculture, Kyushu University, Fukuoka, 812-8581, \\ Japan \\ ${ }^{2}$ Bio-Architecture Center, Kyushu University, Fukuoka, 812-8581, Japan
}

\begin{abstract}
Increased osteoclastic bone resorption plays a central role in the pathogenesis of many bone diseases, and osteoclast inhibitors are the most widely used treatments for these diseases. Ganoderic acid DM, the main component of Ganoderma lucidum, has been known for its medicinal effects such as anti-androgen and anti-proliferative activities. In this study, we investigated the inhibitory effects of ganoderic acid DM and its analog (methyl ganoderic acid DM and 7oxo-methyl ganoderic acid Z) on osteoclastogenesis using RAW264 cell in vitro. Methyl ganoderic acid DM blocked osteoclastogenesis completely at $12.5 \mu \mathrm{M}$ with low cytotoxicity less than $30 \%$. On the other hands, ganoderic acid DM blocked osteoclastogenesis completely at the higher concentration of $50 \mu \mathrm{M}$, but 7-oxo-methyl ganoderic acid $\mathrm{Z}$ did not up to $100 \mu \mathrm{M}$. These results implicated the carbonyl group at C-3 is essentially for selective osteoclastogenesis inhibitory activity, and methyl esters at C-26 should play an important role in enhancing its osteoclastogenesis inhibitory activity.
\end{abstract}

Keyword: Ganoderma lucidum, methyl ganoderic acid DM, osteoporosis, rheumatoid arthritis.

\section{INTRODUCTION}

The balance between bone resorption (by osteoclasts) and bone formation (by osteoblasts) maintains bone homeostasis in a process called bone remodeling [1]. Bone is obviously resistant to dissolution, at least outside the body. Inside the body's highly active milieu, however, bone is remodeled at such a high speed that approximately $10 \%$ of the total bone content is replaced per year in adult humans [2]. Incremental changes in the rate of bone resorption can lead to bone disruption. This striking contrast emphasizes what an extraordinary and specific role osteoclasts play in the active maintenance of the bony skeleton. Large tartrate-resistant acid phosphatase (TRAP)-positive multinucleated cells (MNCs) that are hematopoietic in origin, osteoclasts are capable of resorbing bone [3,4]. These multinucleated cells help dynamically remodel bones in coordination with osteoblasts, which deposit bone matrix. The excessive osteoclastic bone resorption relative to osteoblastic bone formation is often associated with osteopenic diseases including osteoporosis and rheumatoid arthritis. Osteoclastogenesis progresses through multiple stages, including differentiation, fusion, and activation (maturation) regulated by various factors, including cytokines, hormones, and other cells in the bone microenvironment. So the inhibition of osteoclast differentiation also has great clinical implications.

Osteoporosis is a very common disease accompanied by a high level of bone resorption, especially for postmenopausal women. Estrogen treatment, or hormone replacement therapy, is considered by many physicians to be the best

*Address correspondence to this author at the Department of Forest and Forest Products Science, Faculty of Agriculture, Kyushu University, Fukuoka, 812-8581, Japan; Tel: +81-92-642-3002; Fax: +81-92-642-3002; E-mail: shimizu@agr.kyushu-u.ac.jp method to prevent bone loss [5]. However, many women do not tolerate the numerous side effects, or are concerned about the possible increased risk of uterine and/or breast cancer [6-8]. There thus remains a need for highly efficacious anti-resorptive agents with excellent safety and tolerability profiles. Several recent reports whose goal is to identify patterns for preventing osteoporosis through daily diet examined the effects of food components and their bioactive components on bone metabolism [9-10]. We also focused on identifying the lead compound for developmenting inhibitors of osteoclastogenesis among medicinal food stuffs.

The fungus G. lucidum (Reishi, Mannentake, or Lingzhi) has been used for centuries in East Asia to treat various human diseases such as hepatitis, hepatopathy, hypertension, nephritis, bronchitis, and cancers $[11,12]$. Its dried powder was especially popular as a cancer chemotherapy agent in the Imperial Court of ancient China [13]. G. lucidum has been reported to produce many bioactive oxygenated triterpenoids. Up to now, over 120 kinds of triterpenoids have been isolated from G. lucidum and the genus Ganoderma. Some of the triterpenoids such as ganoderic and lucidic acids, isolated from Ganoderma, have demonstrated cytotoxicity against mouse sarcoma and mouse lung carcinoma cells in vitro [14]. As part of our continuing search for biologically active anti-osteoporotic compounds, we found that osteoclast differentiation was inhibited by the ethanol extracts of G. lucidum and ganoderic acid DM (1) which was isolated as one of the active compounds by bioassay-guided fractionation [15]. Ganoderic acid DM (1) especially suppresses the expression of c-Fos and nuclear factor of activated $\mathrm{T}$ cells $\mathrm{c} 1$ (NFATc1). This suppression leads to the inhibition of dendritic cell-specific transmembrane protein (DC-STAMP) expression and reduces osteoclast fusion. These results prompted us to investigate the ability of gano- 
deric acid DM (1) analogs $(\mathbf{2}, \mathbf{3})$ (Fig. 1) to inhibit osteoclast differentiation to determine what structural elements are important for the potent inhibition of osteoclast differentiation.

\section{MATERIALS AND METHOD}

\section{Chemicals}

The chemicals used were Dulbecco's modified Eagle's medium (DMEM) (Sigma, St. Louis, MO, USA), trimethylsilyldiazomethane (Tokyo chemical industry CO. LTD, Japan), activated charcoal, ethylenediaminetetraacetic acid (EDTA), dimethyl sulfoxide (DMSO), WST-1[4-[3-(4iodophenyl)-2-(4-nitrophenyl)-2H-5-tetrazolio]-1,3-benzene disulfonate] (Wako, Osaka, Japan), glutamine (Nissui, Tokyo, Japan), penicillin, streptomycin, and trypsin (Invitrogen, Carlsbad, CA, USA). Fetal bovine serum (FBS), $\alpha-$ MEM was purchased from GIBCO BRL (Grand Island, NY, USA), soluble RANKL (sRANKL) was purchased from PeproTech EC Ltd (London, UK), and TNF- $\alpha$ was obtained from Roche Molecular Biochemical (Mannheim, Germany). Tartrate-resistent acid phosphatase (TRAP) staining kit was purchased from Sigma-Aldrich (St. Louis, MO, USA). Ganoderic acid DM (1) [16]. was available from our previous works. The ${ }^{13} \mathrm{C}$ NMR spectra of methyl ganoderic acid DM (2) and 7-oxo-methyl ganoderic acid Z (3) [17] were measured. The molecular formula of these two compounds were estimated from its liquid chromatographs mass spectral-ion trap-time of flight (LCMS-IT-TOF) spectrum $\left(2:\left[\mathrm{M}+\mathrm{H}^{+}\right]\right.$at $m / z$ 483.3469, calculated for $\mathrm{C}_{31} \mathrm{H}_{46} \mathrm{O}_{4}, 482.3396 ; 3$ : $\left[\mathrm{M}+\mathrm{H}^{+}\right]$ at $m / z 485.3504$, calculated for $\mathrm{C}_{31} \mathrm{H}_{48} \mathrm{O}_{4}, 484.3553$ ).

Methyl ganoderic acid DM (2): ganoderic acid DM (10 $\mathrm{mg})$ in methanol $(1 \mathrm{ml})$-benzene $(3 \mathrm{ml})$ was added to trimethylsilyldiazomethane $(1.5 \mathrm{ml})$ at room temperature. The mixture was stirred for $30 \mathrm{~min}$ at room temperature and concentrated to give the corresponding methyl esters of ganoderic acid DM as a white powder, then followed by preparative HPLC (column: Inertsil ODS-3(20 mm i.d. x 250 $\mathrm{mm}$, GL Science, Inc. USA), methanol: water $=80: 20$, flow rate: $10 \mathrm{ml} / \mathrm{min}$ ) afforded methyl ganoderic acid DM (2) (Rt: $23 \mathrm{~min}$ ). The molecular formula of methyl ganoderic acid DM (2) was determined to be $\mathrm{C}_{31} \mathrm{H}_{46} \mathrm{O}_{4}$ on the basis of the ion peak at $m / z 483.3469\left[\mathrm{M}+\mathrm{H}^{+}\right]$in LCMS-IT-TOF spectrum. The ${ }^{1} \mathrm{H}$ NMR spectrum (Table 1) of 2 indicated the presence of eight methyl singlets at $\delta 0.53,0.78,0.94$, $0.96,1.18,1.68,3.57,6.60$, an olefinic proton triplet at $\delta$ 6.60. The ${ }^{13} \mathrm{C}$ NMR spectrum (Table 1) of 2 showed the presence of eight methyls $(\delta 5.86,12.31,17.87,18.54$, $21.36,24.87,25.32,51.65)$, nine methylenes $(\delta 23.79,25.58$, $34.34,28.54,30.08,31.83,35.33,34.82,37.13)$, three methines $(\delta 36.17,48.93,50.36)$, four quaternary carbons $(\delta 39.38,44.92,47.2,47.76)$, four olefinic carbons $(\delta 127.19$, $139.48,142.97,162.67)$, two ketone carbons ( $\delta 198.03$, $214.63)$, and one carboxyl carbon $(\delta 168.71)$. Compare with the spectrum with 1 , these ${ }^{1} \mathrm{H}$ NMR and ${ }^{13} \mathrm{C}$ NMR data (Table 1) led us to suggest that compound $\mathbf{2}$ was the methyl esters of compound $\mathbf{1}$.

7-Oxo-methyl ganoderic acid Z (3): Conversion of C-3 keton of 2 to C-3 hydroxyl group was carried out by reduction with $\mathrm{NaBH}_{4}$. $\mathrm{NaBH}_{4}(10 \mathrm{mg})$ was added to $2(10 \mathrm{mg})$ in methanol $(3 \mathrm{ml})$ at room temperature. The mixture was stirred for $30 \mathrm{~min}$ at room temperature and the reaction was stopped by added acetic acid $(1 \mathrm{ml})$. The mixture was concentrated in vacuo, then followed by preparative HPLC (column: Inertsil ODS-3, methanol: water $=80: 20$, flow rate: $10 \mathrm{ml} / \mathrm{min}$ ) afforded 7-oxo-methyl ganoderic acid $\mathrm{Z} \mathrm{(3)}$ (Rt: $11 \mathrm{~min}$ ) as a white powder. The molecular formula of 7-oxo-methyl ganoderic acid $\mathrm{Z}(3)$ was determined to be $\mathrm{C}_{31} \mathrm{H}_{48} \mathrm{O}_{4}$ on the basis of the ion peak at $\mathrm{m} / \mathrm{z} 485.3504$ $\left[\mathrm{M}+\mathrm{H}^{+}\right]$in LCMS-IT-TOF spectrum. The ${ }^{13} \mathrm{C}$ NMR spectrum (Table 1) of $\mathbf{3}$ revealed total 31 carbon signals including 8 methyls, 9 methylenes, 4 methines, 4 quatemary carbons, 4 olefinic carbons, 1 carboxyl, 1 ketonecarbonyl, and 1 hydroxyl. Comparison of the ${ }^{13} \mathrm{C}$ chemical shifts of $\mathbf{3}$ with those of $\mathbf{2}$ indicated that the structure of compound $\mathbf{3}$



\section{Cell Cultures}

RAW264 cells were maintained in DMEM with $10 \%$ FBS. All media were supplemented with $2 \mathrm{mM}$ glutamine, $100 \mathrm{IU} / \mathrm{ml}$ penicillin, and $100 \mathrm{mg} / \mathrm{ml}$ streptomycin. Incubations were performed at $37^{\circ} \mathrm{C}$ in $5 \% \mathrm{CO}_{2}$ in humidified air. For osteoclast generation and other experiment, $\alpha$-MEM medium was used.

\section{TRAP-Positive Cell Staining}

RAW264 cells were suspended in phenol $\alpha$-MEM containing $10 \%$ FBS and plated at a concentration of $6.8 \times 10^{3}$ cells/well into a 96-well culture dish in the presence of 30 $\mathrm{ng} / \mathrm{ml} \mathrm{RANKL}$ and TNF- $\alpha(10 \mathrm{ng} / \mathrm{ml})$, then incubated for 24 $\mathrm{h}$ [18]. Then, different concentrations of each compound were added to the cultures. After 3 days of culture, the cells were fixed and stained for TRAP using the TRAP staining kit according to the manufacturer's instructions. TRAP staining cells with more than three nuclei were counted as osteoclast.

\section{WST-1 Assay}

RAW264 cells were suspended in phenol $\alpha$-MEM containing $10 \%$ FBS and plated at a concentration of $6.8 \times 10^{3}$ cells/well into a 96-well culture dish in the presence of 30 $\mathrm{ng} / \mathrm{ml} \mathrm{RANKL}$ and TNF- $\alpha(10 \mathrm{ng} / \mathrm{ml})$, then incubated for 24 $\mathrm{h}$. Then, different concentrations of each compound were added to the cultures. After 3 days of culture, the number of viable cells was compared by WST-1[4-[3-(4-iodophenyl)-2(4-nitrophenyl)-2H-5-tetrazolio]-1,3-benzene disulfonate] assay. Following incubation of cells for the above mentioned time, $10 \%$ WST-1 solution was added to each well and incubated at $37^{\circ} \mathrm{C}$ for three hours. Following incubation, plates were slightly shaken and immediately read at $450 \mathrm{~nm}$ with a scanning multiwell spectrophotometer.

\section{Statistical Analysis}

Data are reported as the mean \pm S.D. Student's t-test for cell experiments was done to determine any significant difference between the groups. Differences between means at the $1 \%$ confidence level $(P<0.01)$ were considered to be statistically significant.

\section{RESULTS}

In our previous work, we found the extremely high potential of the ethanol extracts of G. lucidum as a regulator 
Table 1. NMR Spectral Data for Compounds 2 and 3 in $\mathrm{CDCl}_{3}$

\begin{tabular}{|c|c|c|c|c|}
\hline Position & 2 & 3 & 2 & 3 \\
\hline \multirow[t]{2}{*}{1} & $1.79(1 \mathrm{H}, \mathrm{m})$ & $1.46(1 \mathrm{H}, \mathrm{m})$ & 35.33 & 34.79 \\
\hline & $2.11(1 \mathrm{H}, \mathrm{m})$ & $1.86(1 \mathrm{H}, \mathrm{m})$ & & \\
\hline 2 & $2.70(1 \mathrm{H}, \mathrm{dt} J=12.9,15.9)$ & $1.69(1 \mathrm{H}, \mathrm{m})$ & & \\
\hline 3 & & $3.30(1 \mathrm{H}, \mathrm{dd}, J=5.7,11.5)$ & 214.63 & 77.94 \\
\hline 4 & & & 47.20 & 38.92 \\
\hline 5 & $1.48(1 \mathrm{H}, \mathrm{dd} J=3.2,14.5)$ & $1.63(1 \mathrm{H}, \mathrm{dd}, J=5.4,12.0)$ & 50.36 & 49.82 \\
\hline 7 & & & 198.03 & 199.05 \\
\hline 8 & & & 139.48 & 138.89 \\
\hline 9 & & & 162.67 & 164.78 \\
\hline 10 & & & 39.38 & 39.76 \\
\hline \multirow[t]{2}{*}{11} & $2.36(1 \mathrm{H}, \mathrm{m})$ & $2.35(1 \mathrm{H}, \mathrm{m})$ & 23.79 & 23.64 \\
\hline & & $2.31(1 \mathrm{H}, \mathrm{m})$ & & \\
\hline 12 & $1.79(1 \mathrm{H}, \mathrm{m})$ & $1.82(1 \mathrm{H}, \mathrm{m})$ & 30.08 & 30.12 \\
\hline 16 & $1.69(1 \mathrm{H}, \mathrm{m})$ & $1.35(1 \mathrm{H}, \mathrm{m})$ & & \\
\hline 17 & $2.16(1 \mathrm{H}, \mathrm{m})$ & $1.44(1 \mathrm{H}, \mathrm{m})$ & 48.93 & 48.92 \\
\hline 18 & $0.69(3 \mathrm{H}, \mathrm{s})$ & $0.70(3 \mathrm{H}, \mathrm{s})$ & 15.86 & 15.80 \\
\hline 19 & $1.34(3 \mathrm{H}, \mathrm{s})$ & $1.21(3 \mathrm{H}, \mathrm{s})$ & 17.87 & 18.36 \\
\hline 20 & $1.43(1 \mathrm{H}, \mathrm{m})$ & $1.47(1 \mathrm{H}, \mathrm{m})$ & 36.17 & 36.22 \\
\hline 21 & $0.94 \mathrm{~d}(3 \mathrm{H}, \mathrm{d}, J=6.1)$ & $0.97(3 \mathrm{H}, \mathrm{d}, J=5.7)$ & 18.54 & 18.60 \\
\hline \multirow[t]{2}{*}{22} & $2.48(1 \mathrm{H}, \mathrm{m})$ & $1.58(1 \mathrm{H}, \mathrm{m})$ & 34.82 & 34.88 \\
\hline & & $1.20(1 \mathrm{H}, \mathrm{m})$ & & \\
\hline \multirow[t]{2}{*}{23} & $2.25(1 \mathrm{H}, \mathrm{m})$ & $2.25(1 \mathrm{H}, \mathrm{m})$ & 25.58 & 25.62 \\
\hline & & $2.12(1 \mathrm{H}, \mathrm{m})$ & & \\
\hline 24 & $6.88(1 \mathrm{H}, \mathrm{dd}, J=8.4,7.7)$ & $6.76(1 \mathrm{H}, \mathrm{t}, J=7.0)$ & 142.97 & 143.08 \\
\hline 25 & & & 127.19 & 127.18 \\
\hline 26 & & & 168.71 & 168.71 \\
\hline 27 & $1.84(3 \mathrm{H}, \mathrm{s})$ & $1.82(3 \mathrm{H}, \mathrm{s})$ & 12.31 & 12.34 \\
\hline
\end{tabular}


Table 1. contd....

\begin{tabular}{|c|c|c|c|c|}
\hline \multicolumn{3}{|c|}{$\delta_{H}$} & \multicolumn{2}{|c|}{$\boldsymbol{\delta}_{\mathrm{C}}$} \\
\hline Position & 2 & 3 & 2 & 3 \\
\hline 28 & $0.94(3 \mathrm{H}, \mathrm{s})$ & $0.93(3 \mathrm{H}, \mathrm{s})$ & 24.87 & 24.98 \\
\hline 29 & $1.12(3 \mathrm{H}, \mathrm{s})$ & $1.00(3 \mathrm{H}, \mathrm{s})$ & 25.32 & 27.41 \\
\hline 30 & $1.10(3 \mathrm{H}, \mathrm{s})$ & $0.88(3 \mathrm{H}, \mathrm{s})$ & 21.36 & 15.29 \\
\hline 31 & $3.58(3 \mathrm{H}, \mathrm{s})$ & $3.71(3 \mathrm{H}, \mathrm{s})$ & 51.65 & 51.69 \\
\hline
\end{tabular}

of bone resorption [15]. Bioassay-guided fractionation led us to isolate ganoderic acid DM (1) as an inhibitor of osteoclastogenesis using RAW cells.

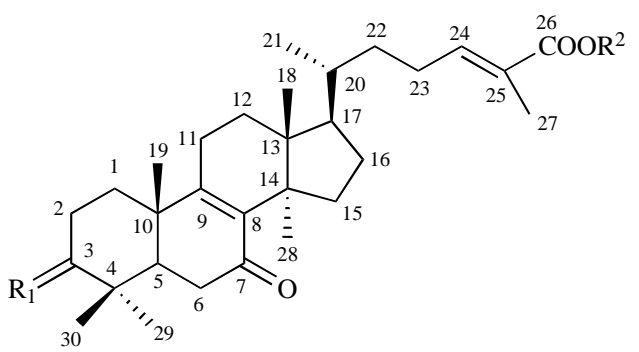

\begin{tabular}{|c|c|c|}
\hline Triterpenoids & $\mathbf{R}^{\mathbf{1}}$ & $\mathbf{R}^{\mathbf{2}}$ \\
\hline \hline Ganoderic acid DM (1) & $\mathrm{O}$ & $\mathrm{H}$ \\
\hline Methyl ganoderic acid DM (2) & $\mathrm{O}$ & $\mathrm{CH}_{3}$ \\
\hline 7-Oxo-methyl ganoderic acid Z (3) & $\widehat{ }^{1 /} \mathrm{H}$ & $\mathrm{CH}_{3}$ \\
\hline
\end{tabular}

Fig. (1). Structures of triterpenoids (1-3).

In general, dissociated form of carboxylic acids such as ganoderic acids is very unlikely to penetrate plasma membrane lipid bilayer. In neutral $\mathrm{pH}$ condition in medium, only small amount of the dissociated form of ganoderic acid DM (1) may enter into cytoplasm of RAW cells revealing the inhibition of osteoclastogenesis. If the specific lanostanemoiety in ganoderic acid DM (1) is essential for inhibition of osteoclastogenesis, but not carboxylic moiety at C-26, the change to the undissociated form of carboxylic moiety, more specifically, methyl ester-form (2) show more potent inhibitory activity than that of $\mathbf{1}$. Furthermore, if so, the structural modification of functional group in lanostane-moiety of $\mathbf{2}$ decreases its inhibitory activity.

Thus, we focused on ganoderic acid DM (1) and its structural related compounds $(2, \mathbf{3})$. To investigate whether these analog affect the differentiation of osteoclasts, we utilized the preosteoclastic cell line, RAW 264 cell, to study the effect of analog $(2,3)$ on the osteoclast-lineage. The cells were treated with each sample for 3 days. The results clearly indicated that not all compounds depressed the osteoclast differentiation (Figs. 2 and 3). Interestingly, among these three compounds, methyl ganoderic acid DM (2) showed the strongest inhibitory activity to osteoclast differentiation than ganoderic acid DM (1) and 7-oxo-methyl ganoderic acid Z (3). Ganoderic acid DM (1) completely blocked the osteoclast differentiation at the concentration of $50 \mu \mathrm{M}$. 7Oxo-methyl ganoderic acid Z (3) showed a low inhibitory activity on osteoclast differentiation even at the high concentration of $100 \mu \mathrm{M}$. Surprisingly, at the concentration of 12.5 $\mu \mathrm{M}$, the osteoclast differentiation was completely blocked by methyl ganoderic acid DM (1). In contrast to methyl ganoderic acid DM (2), ganoderic acid DM (1) and 7-oxo-methyl ganoderic acid Z (3) showed no effect on osteoclast differentiation at this concentration. Almost complete inhibition of differentiation for ganoderic acid DM (1) was observed at 50 $\mu \mathrm{M}$, but still remain $75 \%$ viable cells. The behavior of ganoderic acid DM (1) seemingly indicated that its inhibitory activity of differentiation not completely comes from its cytotoxicity. Like ganoderic acid DM (1), almost complete inhibition of differentiation for methyl ganoderic acid DM (2) was observed at $12.5 \mu \mathrm{M}$, but still remain more than $70 \%$ viable cells observed up to $20 \mu \mathrm{M}$. It should be noted that the limitation of solubility of methyl ganoderic acid DM (2) prevented future investigation more than $25 \mu \mathrm{M}$. Thus, we became aware that the inhibitory activity of methyl ganoderic acid DM (2) on differentiation was caused by not its cytotoxicity, but more like specific inhibition of differentiation. Furthermore, as speculated above, the easier penetration of $\mathbf{2}$ to inside of cells than $\mathbf{1}$ might enhance its inhibitory activity of osteoclastogenesis. This behavior, more specifically, selective inhibitory activity against differentiation, not viability, was important because safety is primarily consideration.

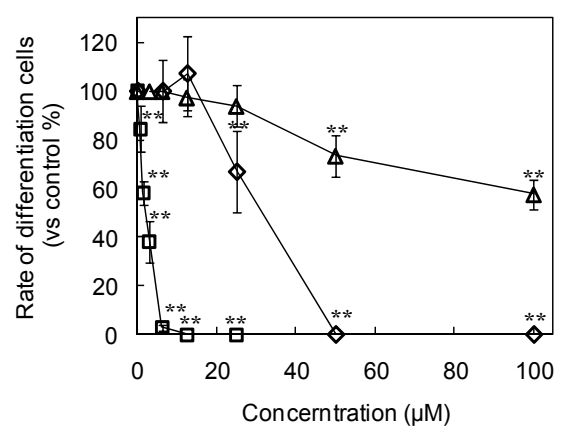

Fig. (2). The effects of ganoderic acid DM (1), methyl ganoderic acid DM (2) and 7-oxo-methyl ganoderic acid Z (3) on the osteoclastogenesis. $(\diamond$ :ganoderic acid DM (1), $\square$ : methyl ganoderic acid DM (2), $\triangle$ : 7-oxo-methyl ganoderic acid Z (3)) Results are given as the mean \pm S.D., $\mathrm{n}=4$. ${ }^{* *} P<0.01$ vs vehicle control. 


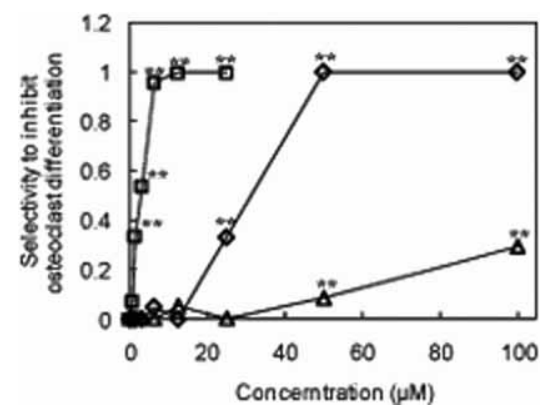

Fig. (3). The effects of ganoderic acid DM (1), methyl ganoderic acid DM (2) and 7-oxo-methyl ganoderic acid Z (3) on selectivity to inhibit the osteoclastogenesis. ( $\diamond$ :ganoderic acid DM (1), $\square$ : methyl ganoderic acid DM (2), $\triangle$ : 7-oxo-methyl ganoderic acid $\mathrm{Z}$ (3)) Results are given as the mean \pm S.D., $\mathrm{n}=4$. ** $P<0.01$ vs vehicle control.

Osteoclast differentiation, in response to receptor activator of NF- $\kappa \mathrm{B}$ ligand (RANKL) and a tumor necrosis factor $\alpha$ (TNF- $\alpha$ ), was inhibited by ganoderic acid DM (1) [15]. The inhibitory activity of ganoderic acid DM is caused by especially suppressing the expression of c-Fos and nuclear factor of activated $\mathrm{T}$ cells $\mathrm{cl}$ (NFATc1). This suppression leads to the inhibition of dendritic cell-specific transmembrane protein (DC-STAMP) expression and reduces osteoclast fusion. Obviously, the mechanistic investigation of osteoclast differentiation inhibitory activity by methyl ganoderic acid DM (1) (Fig. 2) needs to be determined for further application against osteoporosis-related disease.

In Fig. (3), we defined selectivity to inhibit osteoclast differentiation by using the equality. selectivity to inhibit osteoclast differentiation $=($ the rate of cell viability $(\%)-$ the rate of differentiation cell (\%) ) / the rate of cell viability $(\%)$.

When the selectivity to inhibit osteoclast differentiation equal 1 , it means that no osteoclast differentiation cell can be found among the living cells. From Fig. (3), methyl ganoderic acid DM (2) showed the highest selectivity to inhibit osteoclast differentiation than ganoderic acid DM (1) and 7oxo-methyl ganoderic acid Z (3). Methyl ganoderic acid DM (2) showed the value of 1 at $12.5 \mu \mathrm{M}$, ganoderic acid DM (1) showed the same value at $50 \mu \mathrm{M}$, and 7-oxo-methyl ganoderic acid $Z$ (3) cannot give the same value even at 100 $\mu \mathrm{M}$.

\section{DISCUSSION}

Methyl ganoderic acid DM (2) and ganoderic acid DM (1), which showed higher selectivity to inhibit osteoclast differentiation, have a carbonyl group at C-3. In contrast to them, 7-oxo-methyl ganoderic acid $\mathrm{Z}$, which showed low selectivity to inhibit osteoclast differentiation, has a hydroxyl group at C-3. These results suggested that a carbonyl group of the $\mathrm{C}-3$ is essential to elicit the selectivity to inhibit osteoclast differentiation, and a methyl group at C-26 is needed to lower the cytotoxicity to osteoclast among these three compounds.
Osteoclasts appear uniquely adapted to produce the microenvironment and the biochemical milieu that are needed to resorb bone. Most notable of these is the cell membrane-associated protein termed receptor activator of RANKL, which is a member of the TNF family of cytokines. RANKL can then bind to its cognate receptor (RANK) on osteoclast precursors and, enhance the differentiation and fusion of these cells to produce functioning multinucleated osteoclasts. Concomitantly, eliminating the forming of osteoclasts means the bone resorption could be repressed. In this study, we found that methyl ganoderic acid DM (2) showed highly selective inhibition of osteoclastogenesis in RAW cells. Thus makes this compound can be used in osteoclastogenesis therapeutics.

It is not clear if the ingested methyl ganoderic acid DM (2) is absorbed into the biological system through the intestinal tract and delivered to the place where they are needed. The colonic microfloras convert most of these ingested triterpenoids into metabolites that then reach the circulation. Further biological evaluation of these triterpenoids is needed from only one aspect, but from a whole and dynamic perspective.

If the inhibitory activity of osteoclastic differentiation by the methyl ganoderic acid DM (2) from G. lucidum is also producible in human osteoclastic cells, it would be useful in a different sense since osteoclast play a crucial role in the osteopenic diseases including osteoporosis and rheumatoid arthritis.

Altogether our results illustrate the ability of some natural product-derived substances, ganoderic acid DM (1) and its analog (2) to inhibit the osteoclasts differentiation. For this kind of triterpenoids, a carbonyl group of the C-3 is essential to elicit the inhibition of osteoclast differentiation, and a methyl group at C-26 is needed to lower the cytotoxicity to osteoclast. Methyl ganoderic acid DM (2) should be a potential candidate for treating osteopenic diseases, such as osteoporosis and rheumatoid arthritis. Accumulation of the knowledge of the regulatory control of osteoclastogenesis on a molecular basis may provide a more rational and scientific approach to design safe and effective osteoclastogenesis control agents.

\section{REFERENCES}

[1] Manolagas, S.C. Birth and death of bone cells: Basic regulatory mechanisms and implications for the pathogenesis and treatment of osteoporosis. Endocr. Rev., 2000, 21, 115-137.

[2] Alliston, T.; Derynck, R. Interfering with bone remodelling. Nature 2002, 416, 686-687.

[3] Suda, T.; Takahashi, N.; Martin, T.J. Modulation of osteoclast differentiation. Endocr. Rev., 1992, 13, 66-80.

[4] Asagiri, M.; Takayanagi, H. The molecular understanding of osteoclast differentiation. Bone, 2007, 40, 251-264.

[5] Anderson, J.J.; Garner, S.C. The molecular understanding of osteoclast differentiation Phytoestrogens and bone. Baillieres Clin. Endocrinol. Metab., 1998, 12, 543-557.

[6] MacDonald, B.R.; Gowen, M. Emerging therapies in osteoporosis. Best practice \& research. Clin. Rheumatol., 2001, 15, 483-496.

[7] Santell, R.C.; Chang, Y.C.; Nair, M.G.; Helferich, W.G. Dietary genistein exerts estrogenic effects upon the uterus, mammary gland and the hypothalamic/pituitary axis in rats. J. Nutr., 1997, 127 , 263-269.

[8] Scheiber, M.D.; Rebar, R.W. Isoflavones and postmenopausal bone health: a viable alternative to estrogen therapy? Menopause, 1999, $6,233-241$. 
[9] Mühlbauer, R.C.; Li, F. Nutrition: Effect of vegetables on bone metabolism. Nature, 1999, 401, 343-344.

[10] Park, C.K.; Lee, Y.; Chang, E.J.; Lee, M.H.; Yoon, J.H.; Ryu, J.H.; Kim, H.H. Bavachalcone inhibits osteoclast differentiation through suppression of NFATc1 induction by RANKL. Biochem. Pharmacol., 2008, 75, 2175-2182.

[11] Yun, T.K. Update from Asia. Asian studies on cancer chemoprevention. Ann. N. Y. Acad. Sci., 1999, 88, 157-192.

[12] Wasser, S.P.; Weis, A.L. Therapeutic effects of substances occurring in higher Basidiomycetes mushrooms: a modern perspective. Crit. Rev. Immunol., 1999, 19, 65-96.

[13] Mizushina, Y.; Hanashima, L.; Yamaguchi, T.; Takemura, M.; Sugawara, F.; Saneyoshi, M.; Matsukage, A.; Yoshida, S.; Sakaguchi, K. A mushroom fruiting body-inducing substance inhibits activities of replicative DNA polymerases. Biochem. Biophys. Res. Commun., 1998, 249, 17-22.

[14] Min, B.S.; Gao, J.J.; Nakamura, N.; Hattori, M. Triterpenes from the spores of Ganoderma lucidum and their cytotoxicity against
Meth-A and LLC tumor cells. Chem. Pharm. Bull., 2000, 48, 10261033 .

[15] Miyamoto, I.; Liu, J.; Shimizu, K.; Sato, M.; Kukita, A.; Kukita T.; Kondo, R. Regulation of osteoclastogenesis by ganoderic acid DM isolated from Ganoderma lucidum. Eur. J. Pharmacol., 2009, 602, 1-7.

[16] Liu, J.; Kurashiki, K.; Shimizu, K.; Kondo, R. Structure-activity relationship for inhibition of $5 \alpha$-reductase by triterpenoids isolated from Ganoderma lucidum. Bioorg. Med. Chem., 2006, 14, 86548660.

[17] Li, C.; Li Y. M.; Sun H.H. New ganoderic acids, bioactive triterpenoid metabolites from the mushroom Ganoderma lucidum. Nat. Prod. Res., 2006, 20(11), 985-991.

[18] Watanabe, T.; Kukita, T.; Kukita, A.; Wada, N.; Toh, K.; Nagata, K.; Nomiyama, H.; Iijima, T. Direct stimulation of osteoclastogenesis by MIP-1alpha: evidence obtained from studies using RAW264 cell clone highly responsive to RANKL. J. Endocrinol., 2004, 180, 193-101.

(C) Liu et al.; Licensee Bentham Open.

This is an open access article licensed under the terms of the Creative Commons Attribution Non-Commercial License (http://creativecommons.org/licenses/ by-nc/3.0/) which permits unrestricted, non-commercial use, distribution and reproduction in any medium, provided the work is properly cited. 